\title{
Management of rotary-translation in complex endodontic anatomies
}

\author{
Italo Di Giuseppe ${ }^{1 *}$, Enrico Paolo Silla ${ }^{2}$ and Vito Antonio Malagnino ${ }^{3}$ \\ ${ }^{1}$ Private Practitioner in Rome, Italy \\ ${ }^{2}$ Catholic University of Sacred Heart, Rome, Italy \\ ${ }^{3}$ University of Chieti, Italy
}

\section{Introduction}

Various endodontic instruments and techniques have been proposed for cleaning and shaping root canal systems. However, the creation of a continuously tapering conical form, the maintenance of the original canal shape and position of the root canal and apical foramen are still difficult, not always predictable, especially in curved canals [1]. In fact, the morphology and the curvatures of the root canal systems vary significantly and these factors provide great challenges for clinicians [2]. Severe apical curvatures, or even double ones, are obviously a stressful and dangerous anatomy for $\mathrm{Ni}-\mathrm{Ti}$ rotary instruments, with an increased risk of intra-canal failure; not only separations, but ledges too. Furthermore, apical abrupt curvatures are not always visible radiographically, but can be encountered very often, especially when two canals join [3].

Root curvature is a frequent occurrence in the human dentition, being one of the various anatomical complexities characterizing the root canal system, and introduces factors that, if not properly controlled during canal preparation, may lead to undesirable technical results [4]. The parameters radius and angle of curvature, as defined by Pruett, et al. [5], are generally employed to describe the geometrical characteristics of curved root canals. The radius of curvature describes, for canals with the same angle of curvature, how abrupt is the canal deviation from a straight line: the smaller the curvature radius, the more abrupt the canal deviation. Abrupt curvatures, especially those localized in the apical one-third, present the greatest difficulty because they do not allow much alteration of the curvature radius via canal coronal enlargement, since their influence is expressed through a very short lever arm [6]. On the other hand, the damage done in an instrument depends on the root canal shape, which is inherent to the canal considered and can be irregular. Indeed, the amount of strain in the file depends directly on the geometry of the root canal [7]. Therefore, when an endodontic $\mathrm{Ni}$-Ti rotary instrument has to shape root canals with severe or double curvature, it encounters high difficulties. In fact, in these particular situations, the phenomenon of rotary-translation stresses endodontic instruments through torsional fatigue, making very difficult to obtain optimal results [8]. In this paper we report examples of teeth with particularly abrupt curvatures, to explain what is the behavior of an endodontic instrument in similar cases and to propose a clinical protocol, in order to manage the difficulties the clinician could face in everyday practice.

\section{Material and methods}

Our approach, when we have to face a root canal with a severe curvature, is the following. It is important to recognize in advance the peculiarity of the anatomy of root canal system, in order to adopt the correct strategy to clean and shape every part of the canals. First of all, we have to obtain a perfect isolation of the tooth and open the pulp chamber trying to save as much tooth tissue is possible, but so as we can properly locate root canals. Then, we use a SS instrument not trying to get to the apex, but just to make a scouting of the canal and get a preliminary working length $(\mathrm{WL})$. When we feel the first resistance into the canal, we abandon the manual instrument and start with the mechanical instrumentation, up to the preliminary WL just established with SS K file \#10. Then, we go back to the SS K file (\#08, \#10), now in order to arrive to the apical foramen. If it is possible, we have got a glide path for the mechanical finishing ready to come. In this phase, it is advisable to use Ni-Ti rotary instruments with very low speed values $(150 \mathrm{rpm})$, maintaining high values of torque. The reason of the importance of working with low speed is the possibility to bypass the effect of the phenomenon of rotary-translation on mechanical instruments. In fact, the more we maintain a low speed rotation, the more rotary-translation's effects are minimal, allowing instruments to go forward, before binding dentin walls. Instead, if with an SS file, after an early enlargement of both coronal and medium one third, we are not able to easily gain the apex, we have to give up. Probably, in these situations, there is a second apical curvature, thus the possibility to make a mistake using SS files. This is the reason why, in these cases, we rotate $\mathrm{Ni}$ - $\mathrm{Ti}$ instruments manually, in order to ultimate root canal shaping up to the foramen. In fact, using Ni-Ti rotary instruments manually, we apply minimum values of speed, anticipating rotary translation and reducing instruments' impact on root canal's walls. So, preventing friction due to impact, we reduce one of the reasons which contrast instruments' advancement. We report some examples of our just mentioned clinical approach. As we can see from the preliminary endo-oral radiography, this is a case of a first mandibular premolar, which presents a particularly abrupt curvature, going mesial to distal, in its apical one-third (Figure 1); first of all, after eliminating the calcification in the coronal one third, we sound the canal with an SS K file \#10, but it can't reach the apex because of the important root canal's radius of curvature. So, after a preliminary mechanical

Correspondence to: Italo Di Giuseppe, Private Practitioner in Rome, Italy, E-mail: studiodigiuseppe2@virgilio.it

Key words: $\mathrm{Ni}$-Ti rotary instruments, rotary translation, torsional fatigue

Received: January 01, 2017; Accepted: January 09, 2017; Published: January 12, 2017 
enlargement, using low speed value (150 rpm), we start to use $\mathrm{Ni}$ $\mathrm{Ti}$ rotary instruments manually rotated, in order to overcome the anatomical complexity. Now, as we can see in the following endo-oral radiography, a Ni-Ti rotary instrument 15.05 taper (manually rotated), can go through the curvature up to the WL (Figure 2). After completing the mechanical instrumentation, we make endo-oral radiography with the corresponding carrier-based obturator inserted in the canal (Figure 3). Now, the double-curvature present in the apical one-third is completely filled. Finally, in the last endo-oral radiography, we report the complete coronal restauration, and how the periapical translucency vanished (Figure 4). With this clinical protocol we are able to clean, both with mechanical instrumentation and irrigation, and shape a very complex root canal such the one reported is. Another example of our clinical approach is that of the first maxillary molar represented in preliminary endo-oral radiography (Figure 5). Since from the preliminary endo-oral radiography we can see that also this tooth presents a severe curvature, going mesial to distal, in the apical onethird of its Mesial-Buccal (MB) root. The clinical protocol adopted is the same as the previous case and, after an initial difficulty, it is possible

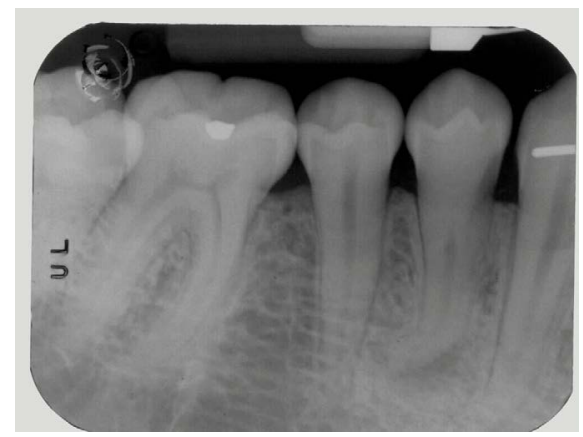

Figure 1. Shows the preliminary image of a first mandibular premolar with a severe apical curvature.

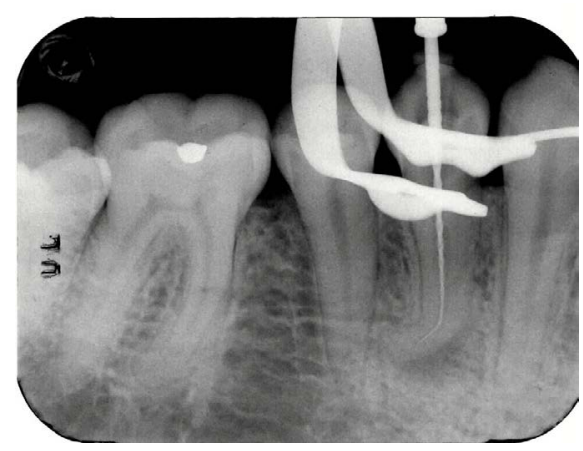

Figure 2. We can see a Ni-Ti rotary instrument, \#15.05 taper, manually driven up to the working length.

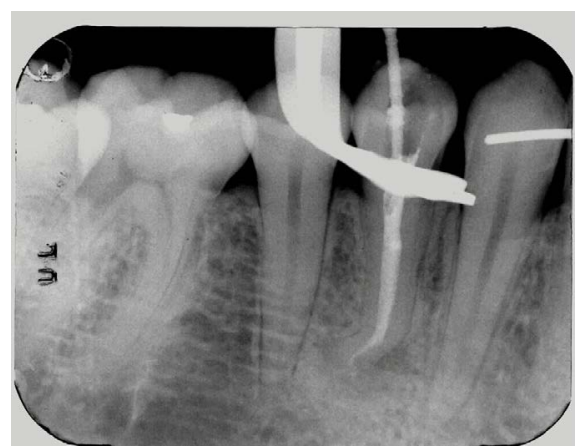

Figure 3. Root canal filled. Note the apical double-curvature. to clean and shape even the MB root in order to completely fill the root canal. In fact, we can observe the corresponding gutta-percha cones inserted in each canal (Figure 6). As we can see, the GP cone fitting the $\mathrm{MB}$ root canal has perfectly reached the working length and we are now ready to fill the root canal system (Figure 7). Furthermore, we can observe another case of a second maxillary premolar with a particular root canal anatomy (Figure 8). In case like this, it is very difficult to reach working length with a SS manual instrument. In fact, we proceed with a mechanical Ni-Ti instrumentation with very low values of speed, and we are able to go through the entire length of root canal, finishing strictly manually (Figure 9). Finally, root canal filling indicates the quality of this approach, effective and respectful of root canal anatomy (Figure 10).

\section{Discussion and conclusion}

As we know from the findings of other authors [9-13] root canal geometry determines the fatigue behavior of $\mathrm{Ni}$-Ti rotary instruments in clinical use, because the stress level they reach depend on the curvature radius of the canal and on the diameter of the file at its maximum bending point. Increased severity in the angle and radius of

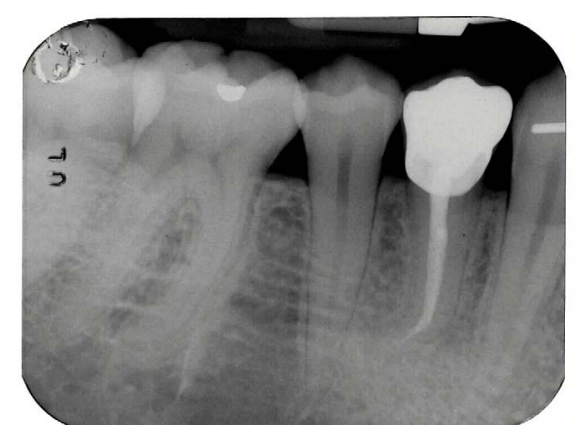

Figure 4. There is the tooth after coronal restauration.

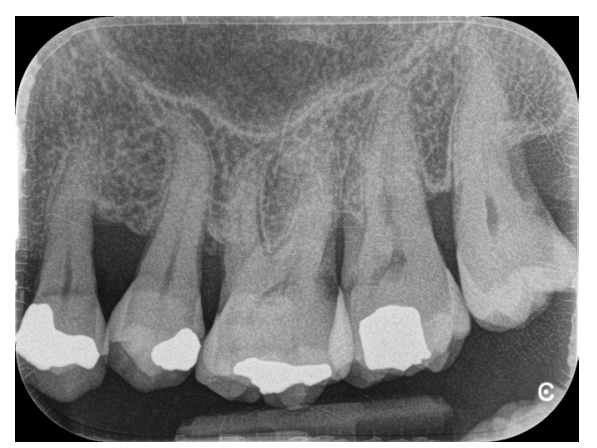

Figure 5. Represents the preliminary radiography of a first maxillary molar.

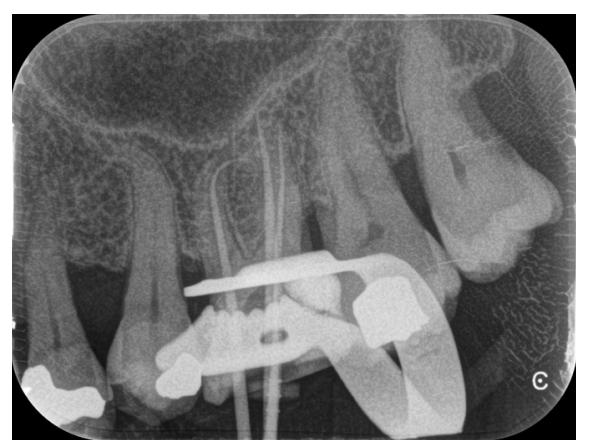

Figure 6. Shows the proof of gutta-percha cones. 


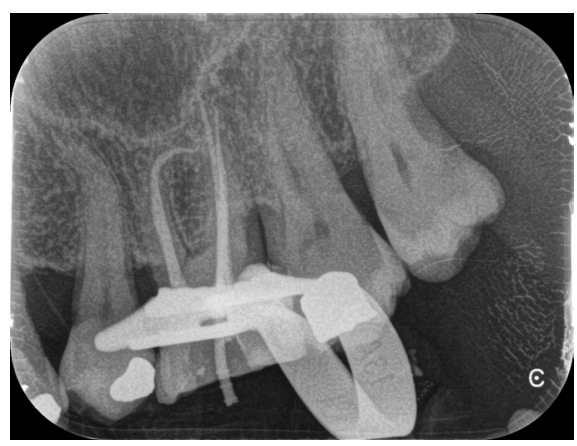

Figure 7. We can see the complete filling of root canal system.

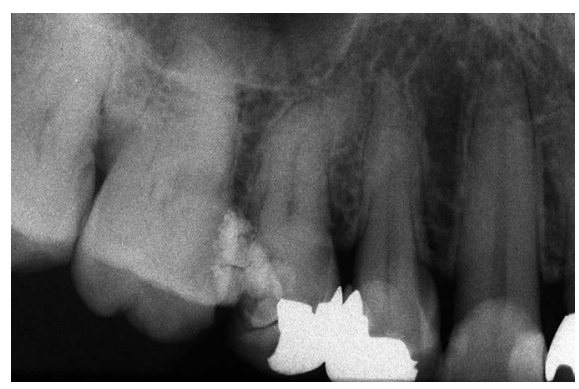

Figure 8. Preliminary endo-oral radiography of a maxillary second premolar.

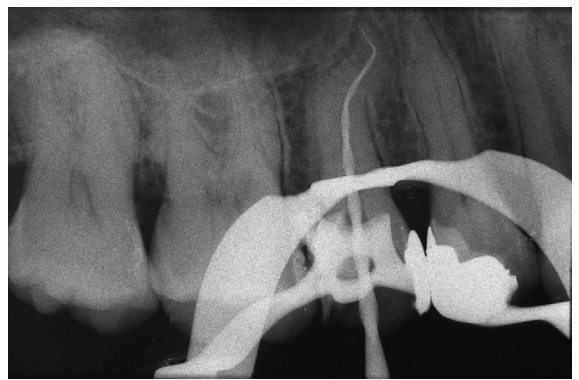

Figure 9. Ni-Ti rotary instrument inserted into root canal up to working length.

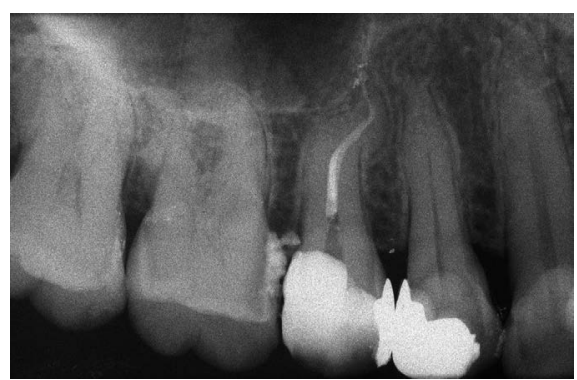

Figure 10. Final endo-oral radiography, showing complete root filling.

the curves around which the instrument rotates decreases instrument lifespan $[5,9,14]$.

Apical abrupt curvatures of root canals represent difficult areas for instrumentation and filling. Little information on the mechanical performance of $\mathrm{Ni}$-Ti rotary instruments in apical abrupt curvatures is available. Furthermore, no evidence has been presented that proves how safe is the use of rotary instruments in such cases. Frequently, abrupt curvatures may be encountered clinically and they may not be visible on $2 \mathrm{D}$ rx. If clinicians become aware of the problem whilst scouting canals, they can take into consideration the use of a manual instrument. If not, it would probably be an advantage to select rotary instruments that resist fracture longer in such complex curvatures [3].

As reported in our previous work [8] the effect of rotary-translation opposes to the progression of endodontic instruments, in particular when there is a curvature. If the curvature is minimal, and the radius of curvature is not particularly strict, this phenomenon becomes quite negligible. While, if the radius of curvature is more prominent, the effect of rotary-translation increases, as well as friction and torsion.

$\mathrm{Ni}$-Ti rotary instruments must be so flexible to permit the clinician to use them safely even in those unexpected apical abrupt curvatures. Both cross-sectional area and file design (influencing stress distribution during load) may affect an instrument's resistance to fracture when subjected to flexural and torsional load [3]. Larger tapered $\mathrm{Ni}-\mathrm{Ti}$ rotary endodontic files should not be considered to be safer or more resistant during clinical use. In fact, these files, when employed to shape curved root canals, will fracture after fewer stress cycles than the smaller tapered ones. This observation suggests that an early discard of the larger tapered instruments may ensure greater efficiency and safety in clinical procedures [4]. This is due to the fact that when an instrument rotates in a curved root canal, any points within it in the segment subjected to the maximum stress, except those in the center (neutral axis), are subjected to repeated tensile or compressive strains. The farther away from the central axis, the greater the imposed strain at that point [15]. This explains why instruments of a larger diameter are affected by fatigue more than smaller ones [16].

In conclusion, we have seen how many difficulties every clinical operator can encounter during the instrumentation of a particularly abrupt root canal, and how easy is to cause a fracture. So, we provide some simple and fundamental advices, in order to manage even the most difficult situation.

\section{Acknowledgments}

The authors deny any conflict of interest related to this study.

\section{References}

1. Schilder H (1974) Cleaning and shaping the root canal. Dent Clin North Am 18: 269296. [Crossref]

2. Cohen S, Burns RC (2001) Pathways of the Pulp, $8^{\text {th }}$ ed., Elsevier Health Sciences.

3. Plotino G, Grande NM, Melo MC, Bahia MG, Testarelli L, et al. (2010) Cyclic fatigue of NiTi rotary instruments in a simulated abrupt curvature. Int Endod J 43: 226-230. [Crossref]

4. Bahia MG, Buono VT (2005) Decrease in the fatigue resistance of nickel-titanium rotary instruments after clinical use in curved root canals. Oral Surg Oral Med Oral Pathol Oral Radiol Endod 100: 249-255. [Crossref]

5. Pruett J, Clement D, Carnes DJ (1997) Cyclic fatigue testing of nickel-titanium endodontic instruments. J Endod 23: 77-85. [Crossref]

6. Roane JB, Sabala CL, Duncanson MG (1985) The "balanced force" concept for instrumentation of curved canals. J Endod 11: 203-211. [Crossref]

7. Viana Ac, Gonzalez BM, Buono VT, Bahia MG (2006) Influence of sterilization on mechanical properties and fatigue resistance of nickel-titanium rotary endodontic instruments. Int Endod J 39: 709-715. [Crossref]

8. Di Giuseppe I, Di Giuseppe D, Malagnino VA, Silla EP, Somma F (2015) Conditioning of root canal anatomy on static and dynamics of nickel-titanium rotary instruments. $G$ Ital Endod 29: 58-64.

9. Haikel Y, Serfaty R, Batema G, Senger B, Allemann C (1999) Dynamic and cyclic fatigue of engine-driven rotary nickel-titanium endodontic instruments. $J$ Endod 25: 434-440. [Crossref]

10. Yared GM, Bou Dagher FE, Machtou P (1999) Cyclic fatigue of Profile rotary instruments after simulated clinical use. Int Endod J 32: 115-119. [Crossref]

11. Yared GM, Bou Dagher FE, Machtou P (2000) Cyclic fatigue of Profile rotary 
Di Giuseppe I (2017) Management of rotary-translation in complex endodontic anatomies

instruments after clinical use. Int Endod J 33: 204-207. [Crossref]

12. Gambarini G (2001) Cyclic fatigue of nickel-titanium rotary instruments after clinical use with low- and high torque endodontic motors. J Endod 27: 772-774. [Crossref]

13. Melo MCC, Bahia MGA, Buono VTL (2002) Fatigue resistance of engine-driven rotary nickel-titanium endodontic instruments. J Endod 28: 765-769. [Crossref]

14. Mize SB, Clement DJ, Pruett JP, Carnes DL Jr (1998) Effect of sterilization on cyclic fatigue of rotary nickel-titanium endodontic instruments. J Endod 24: 843-847. [Crossref]

15. Craig RG. Restorative Dental Materials, $10^{\text {th }}$ edn. St Louis, MO, USA: Mosby.

16. Plotino G, Grande NM, Sorci E, Malagnino VA, Somma F (2006) A comparison of cyclic fatigue between used ans new Mtwo Ni-Ti rotary instruments. Int Endod J 39: 716-723. [Crossref]

Copyright: (C2016 Di Giuseppe I. This is an open-access article distributed under the terms of the Creative Commons Attribution License, which permits unrestricted use, distribution, and reproduction in any medium, provided the original author and source are credited. 